Metzler Musik Chronik 


\title{
METZLER MUSIK CHRONIK
}

\author{
vom frühen Mittelalter \\ bis zur Gegenwart
}

von Arnold Feil

Verlag J. B. Metzler

Stuttgart - Weimar 
Die Deutsche Bibliothek - CIP-Einheitsaufnahme

\section{Feil, Arnold:}

Metzler Musik Chronik vom frühen Mittelalter bis zur Gegenwart von Arnold Feil. - Stuttgart ; Weimar : Metzler, 1993

$$
\text { ISBN 978-3-476-00929-6 }
$$

NE: HST

$$
\begin{gathered}
\text { ISBN 978-3-476-00929-6 } \\
\text { ISBN 978-3-476-03481-6 (eBook) } \\
\text { DOI 10.1007/978-3-476-03481-6 }
\end{gathered}
$$

Dieses Werk einschließlich aller seiner Teile ist urheberrechtlich geschützt. Jede Verwertung außerhalb der engen Grenzen des Urheberrechtsgesetzes ist ohne Zustimmung des Verlages unzulässig und strafbar. Das gilt insbesondere für Vervielfältigungen, Übersetzungen, Mikroverfilmungen und die Einspeicherung und Verarbeitung in elektronischen Systemen.

$$
\text { (C) } 1993 \text { Springer-Verlag GmbH Deutschland }
$$

Ursprünglich erschienen bei J. B. Metzlersche Verlagsbuchhandlung und Carl Ernst Poeschel Verlag GmbH in Stuttgart 1993 
Dem Andenken

der Eltern

und der Lehrer

Else Rehberg (1900-1970)

Siegfried Hermelink (1914-1975)

Thrasybulos G. Georgiades (1907-1977)

Fritz Ernst (1905-1963)

Walther Bulst (1899-1986)

Ewald Jammers (1897-1981)

Walter Gerstenberg (1904-1988)

Sol Babitz (1911-1982)

und für Heinrich Buhr 


\section{Inhaltsverzeichnis}

Vorwort

\section{ZWISCHEN ANTIKE UND MitTELALTER}

I. Zur Musik des nördlichen Europas zwischen Antike und Mittelalter

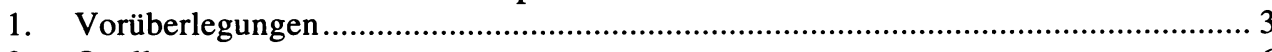

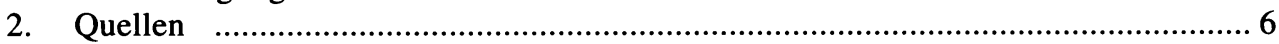

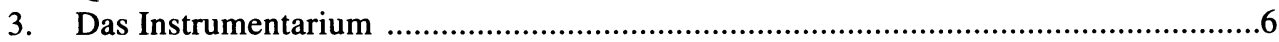

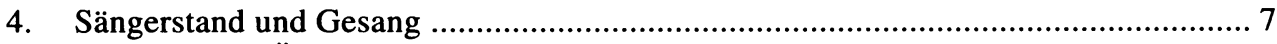

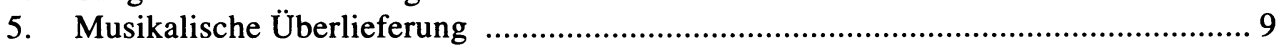

\section{MitTelalteR}

II. Die Liturgie der Kirche und die einstimmige liturgische Musik

1. Kurze Geschichte der Liturgie und der liturgischen Musik des christlichen Gottesdienstes bis ins Mittelalter ............................................. 15

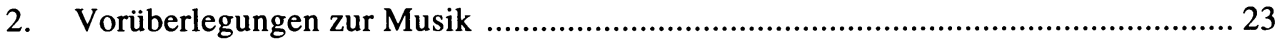

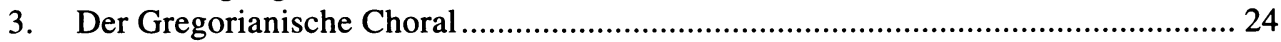

III. Die Entstehung der Polyphonie: 800 bis 1430

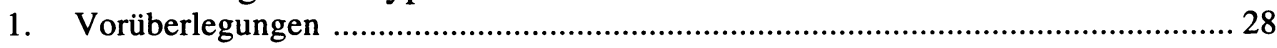

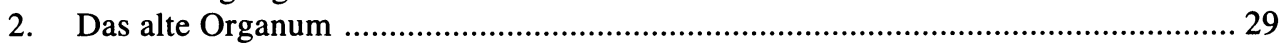

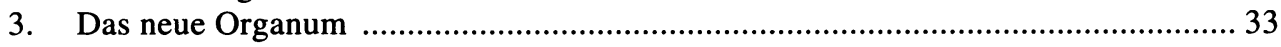

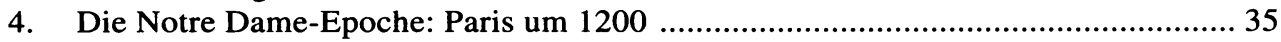

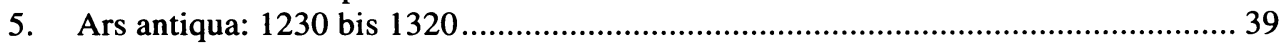

6. Ars nova: das 14. Jahrhundert in Frankreich ................................................ 44

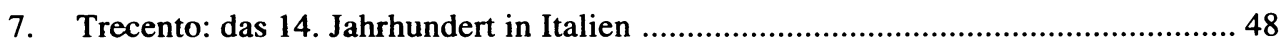

8. Das späte 14. Jahrhundert in Frankreich und Italien .........................................49

9. Das 14. und das beginnende 15. Jahrhundert in England und in Deutschland

IV. Die Ausbildung der Vokalpolyphonie im Niederländischen Zeitalter der Musik: 1430 bis etwa 1550

1. Überlegungen zur Renaissance in der Musik ……………………..................... 51

2. Gruppierungen der Musiker und der Überlieferung ............................................ 51

3. Von der sogenannten Burgundischen Epoche zum 16. Jahrhundert ........................ 52

4. Die nationalen Komponenten in der Musik der ersten Hälfte des 16. Jahrhunderts .................................................................................... 57

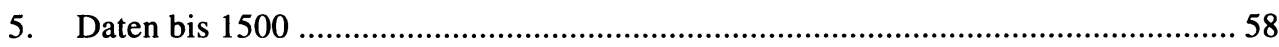

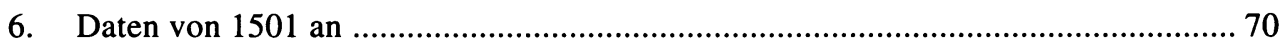




\section{RENAISSANCE}

V. Die Blütezeit der klassischen a cappella-Polyphonie:

die 2. Hälfte des 16. Jahrhunderts

1. Das Werden der abendländischen Polyphonie »am Ziel « ..........................................8 87

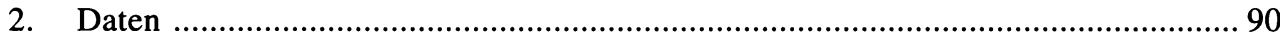

VI. Das Aufstreben der Instrumentalmusik zur polyphonen

Komposition und die erste Epoche einer selbständigen

komponierten Instrumentalmusik: 1430 bis 1640

1. Zur sogenannten ältesten Instrumentalmusik

2. Grundsätzliche Überlegungen zu den Bedingungen des musikalisch Instrumentalen und zu den Konsequenzen

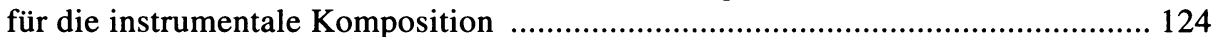

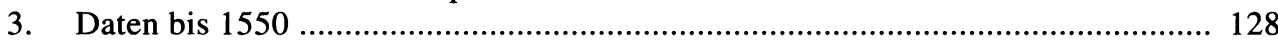

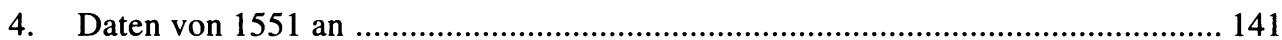

\section{BAROCK}

VII. Das Zeitalter der harmonischen Polyphonie oder

Das Generalbaßzeitalter: 1600 bis 1750

1. Die neue Verbindung von »vokal « und »instrumental « und der »harmonische Kontrapunkt« im sogenannten Generalbaßsatz

2. Das Zeitalter der Monodie und der Oper

a) Die Oper ein Renaissance-Phänomen?

b) Die Oper als Teil des höfischen Festes des Barocks ......................................... 166

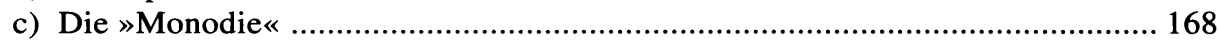

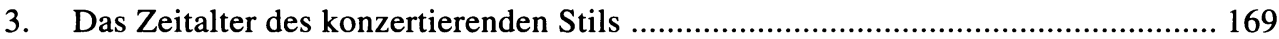

4. Das italienische Zeitalter der Musik und das Zeitalter der deutschen evangelischen Kirchenmusik ...................................................... 171

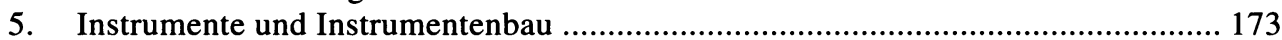

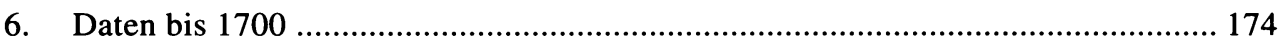

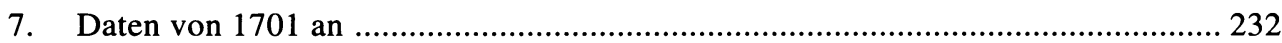

\section{DIE MUSIKALISCHE FRÜHROMANTIK}

VIII. Das Ende des Zeitalters der Polyphonie und der Übergang zu einer Musik mit neuem Interesse: 1730 bis 1820

1. Überlegungen zur Bedeutung und zur Benennung dieser Epoche und zur Epochenbezeichnung »Frühromantik « statt »Vorklassik«

2. Die Musik im Dienste eines neuen Ausdrucksbedürfnisses des Menschen für seine Empfindungen; die Musik als »Tonsprache«: erste romantische Züge in der Musik

3. Die Verschiebung der sozialen Grundlagen des Musiklebens und der Musik im beginnenden Industriezeitalter

4. Die musikalischen Folgen dieser Verschiebungen

5. Die wichtigsten Musikstädte und musikalischen »Schulen « in der 2. Hälfte des 18. Jahrhunderts

6. Daten 


\section{DIE WIENER KLASSIKER}

IX. Haydn, Mozart und Beethoven - und Franz Schubert:

$1755 / 1781$ bis 1828

1. Überlegungen zur Epochenbezeichnung "Klassik « ............................................... 371

2. $\quad$ Was eigentlich ist romantisch? ...................................................................... 372

3. Die Grundintention des Musikalischen, gedacht bei Schiller und bei seinem

Freund und Schüler Christian Gottfried Körner ................................................... 373

4. Beispiel: Mozart, Le nozze di Figaro, Arie des Figaro »Non più andrai« .............. 375

5. Beispiel: Beethoven, 3. Klavierkonzert c-moll op. 37, Erster Satz ....................... 378

6. Die Grundintention des Musikalischen bei Haydn, Mozart und Beethoven .......... 379

7. ... und bei Franz Schubert: »Der Klassiker Schubert « ........................................... 379

8. Weitere Überlegungen zur Epochenbezeichnung »Klassik « .................................. 382

9. Das musikalische Satzprinzip des »obligaten Accompagnements « und das Durchführungsprinzip der »Sonate « ....................................................... 383

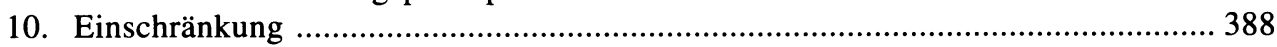

11. Die Klaviermusik der Wiener Klassiker (von August Gerstmeier) ........................ 389

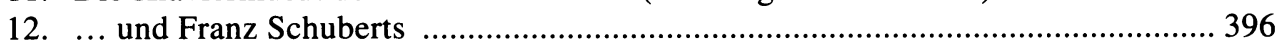

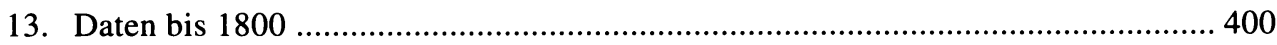

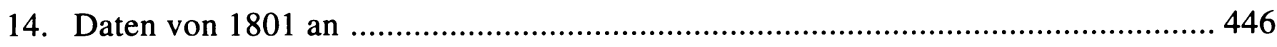

\section{DIE MUSIKALISCHE HOCHROMANTIK}

X. Das 19. und das beginnende 20. Jahrhundert: etwa 1820 bis etwa 1920

1. Überlegungen zur Epochenbezeichnung »Romantik « für die Musikgeschichte .... 501

2. ... und zu Franz Schubert: „Der Romantiker Schubert « ....................................... 502

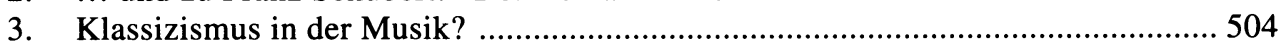

4. Zur Entstehung musikalischer Nationaldiome …………................................... 505

5. Das Genie und die Vielen ................................................................................. 507

6. Der autonome Künstler und das autonome Kunstwerk ......................................508

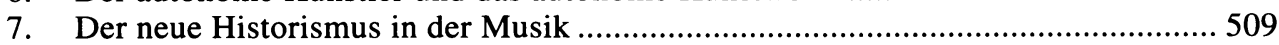

8. Die neue Musikästhetik des 19. Jahrhunderts ...................................................... 510

9. Der neue Musiker: Der Interpret ……………........................................... 511

10. Der neue Musikbetrieb ................................................................................ 512

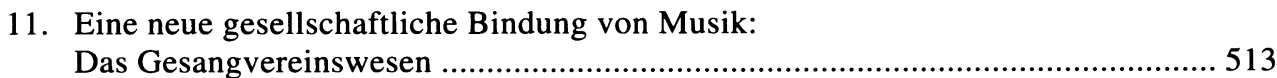

12. Das neue musikalische Phänomen Unterhaltungsmusik ....................................... 514

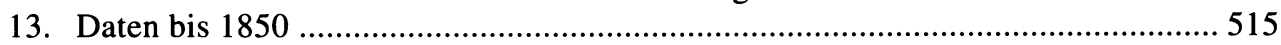

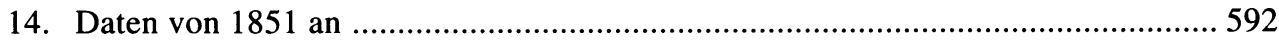

\section{MODERNE MUSIK}

XI. Musik zwischen etwa 1890 und etwa 1920

1. Überlegungen zur »Moderne« und zu »Realismus«, »Verismus« und »Impressionismus « in der Musik

2. Zum musikalischen »Expressionismus « ...............................................................65

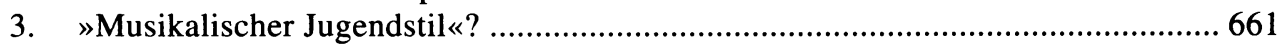

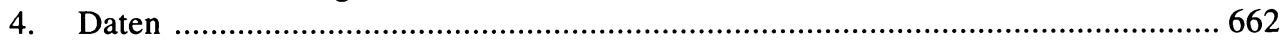




\section{NEUE MuSIK}

XII. Musik zwischen etwa 1910 und etwa 1950

1. Vorbemerkungen: Zur geistigen Situation der Zeit für die musikalische

Komposition - und zu den Konsequenzen daraus für Produktion und

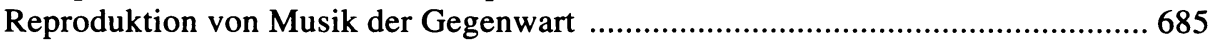

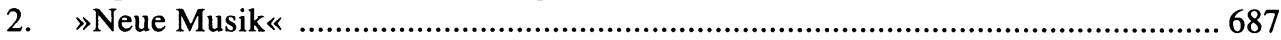

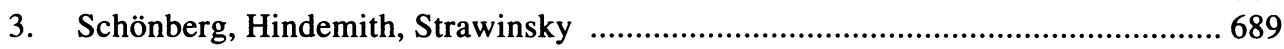

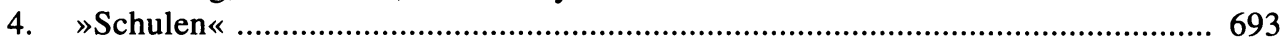

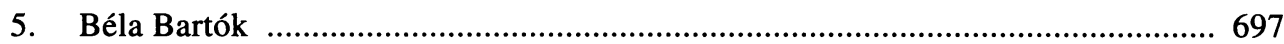

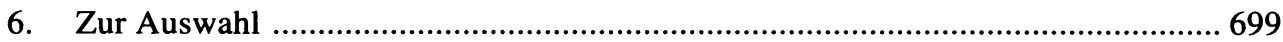

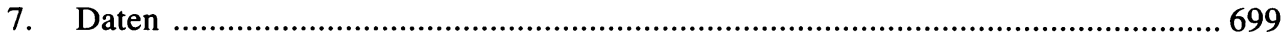

\section{MusiK IN DER GegenwART}

XIII. Die Musik im Zeitalter neuer technischer Möglichkeiten:

Musik seit etwa 1950

1. Schallplatte, Lautsprecher und Tonband und ihre Folgen für das Hören, das Musikhören, das Musikmachen und für die musikalische Komposition ......... 747

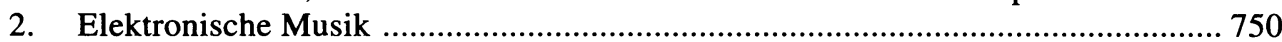

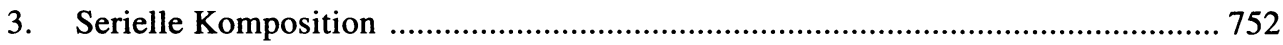

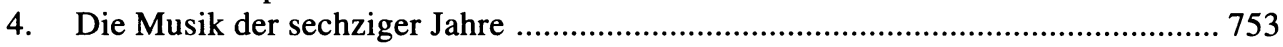

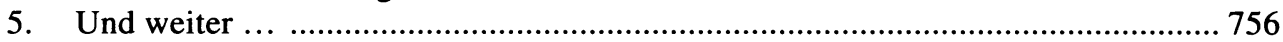

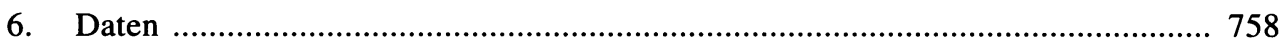

XIV. Musik alsGeschichte

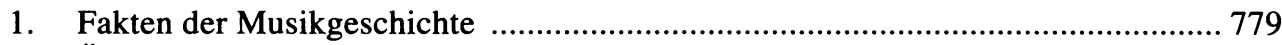

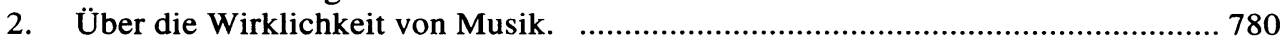

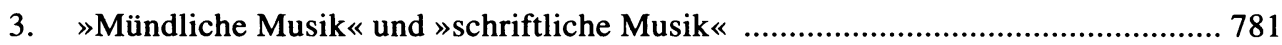

4. »Entwicklung « in der Musikgeschichte? .............................................................. 783

5. Das Problem der Epochengliederung in der Musikgeschichte .............................. 783

6. Die Geschichte und ihre Quellen. Das Problem der Bewertung

7. Auftrag und Entstehung des einzelnen Werkes und die Bedingungen für die Entstehung besonderer Gattungen als Gegenstände

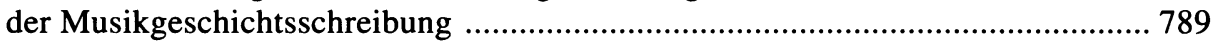

8. Einschränkungen ........................................................................................ 790

NACHWORT

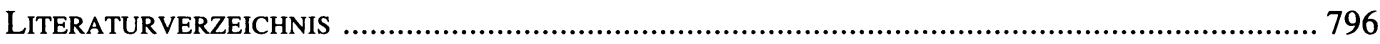

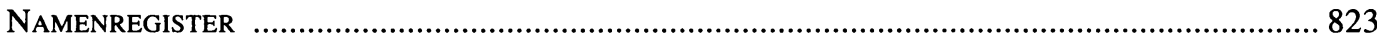




\section{Inhaltsverzeichnis chronologisch}

um 360 Hilarius von Poitiers: Liber Hymnorum 16

386 Ambrosius von Mailand: Hymnen

vor 395 Augustinus: Psalmus contra partem Donati

nach 496 Chlodwig I. tritt zum Christentum über

nach 500 Benedikt von Nursia:

Benediktinerordensregel

um 600 Papst Gregor der Große: Reform der Liturgie

719 Papst Gregor II. beauftragt den Benediktiner Winfrid/Bonifatius mit der Missionierung Deutschlands

785/786 Papst Hadrian I. schickt ein Sacramentar ins Frankenreich

um 790 Karl der Große holt Mönche aus Rom um 800 Alcuin: Lehrbuch der Artes Liberales 800-1430 Entstehung der Polyphonie um 850 Aurelianus Reomensis: Musica disciplina

2. Hälfte 9. Jahrhundert Musica Enchiriadis und ihre Traktatgruppe

984-94 Orgel in der Kathedrale von Winchester/England

Anfang 11. Jahrhundert Winchester Tropar

1025/26 Guido von Arezzo: Micrologus

um 1100 Mailänder Traktat »Ad organum faciendum "

12. Jahrhundert St. Martial-Schule

Anfang 12. Jahrhundert Johannes Cotto

um 1140 Codex Calixtinus

1160/80-1230/50 Notre Dame-Schule

vor 1200 Leoninus: Magnus liber organi

um 1200 Perotinus Magnus

1230-1320 Ars antiqua

2. Viertel 13. Jahrhundert Vatikanischer Organumtraktat

14. Jahrhundert in Frankreich: Musik der Ars nova

— in Italien: Musik des »Trecento

- Robertsbridge-Fragment

1309-76 Papstresidenz in Avignon

um 1316 Roman de Fauvel

nach 1320 Philippe de Vitry:

Notationstraktat "Ars nova"

1321 Johannes de Muris: Musiktraktat

1324/25 Papst Johannes XXII.: Bulle zur

Kirchenmusik

1364 Machaut: Messe zur Krönung des

französischen Königs in Reims

1377 Doppelballade auf den Tod von Machaut
Das späte 14. Jahrhundert in Frankreich und Italien

Das 14. und das beginnende 15. Jahrhundert in England und in Deutschland

1. Hälfte 15. Jahrhundert: Burgundische Epoche

1417 Tapissier: Motette »Eya dulcis» 58

um 1420 Instrumentalmusik im Codex Faenza 128

1426 Dufay: Motette »Apostolo glorioso" 58

um 1426-1428 Dufay: Missa "Sancti Jacobi« 58

um 1430 Dufay: Hymnen-Zyklus per totum annum

- Dufay: Chanson "Helas, ma dame par amours"

1430 Binchois wird Mitglied der burgundischen Hofkapelle

1430-etwa 1550 Niederländisches Zeitalter 51

1433 Dufay: Motette »Supremum est mortalium bonum"

1436 Dufay: Domweih-Motette für Florenz 60

um 1440 Binchois: Chansons

1440 Martin Le Franc: Le Champion des Dames

um 1440-um 1480 Trienter Codices

1448 Tabulatur des Adam Ileborgh

um 1450 Dufay und Ockeghem: Missae

"L'homme armé"

1450-70 Buxheimer Orgelbuch

1452 Paumann: Fundamentum organisandi 130

1452-60 Lochamer Liederbuch

1453 Dufay: Klagemotette zum „Fasanenbankett" um 1470 Ockeghem: Missa cuiusvis toni

- Ockeghem: Missa Mi-Mi

- Ockeghem: Missa Prolationum

- Ockeghem: Fuga trium vocum

nach 1470 "Aulen-Messe"

$1472 / 73$ Johannes Tinctoris: Terminorum Musicae Diffinitorium

um 1474 Tinctoris: Proportionale musices

um 1475 Busnois: Missae

um 1475-1480 Canti carnascialeschi

1477 Tinctoris: Liber de arte contrapuncti

um 1480 Glogauer Lieder- und Musikbuch

- Guilelmus Monachus: De praeceptis artis musicae

- Obrecht und Josquin: Missae "Fortuna desperata"

um 1480 und 1505 Ockeghem und Obrecht: Missae »Caput»

1484/87 Compère und Obrecht: Motetten "Quis numerare queat" 
1485-um 1500 Mensuralcodex Berlin 40021 um 1490 Heinrich Finck, Adam von Fulda und andere: Mehrstimmige Hymnen 1490 Adam von Fulda: De Musica 1492-1500 Gaffurius: Trilogia Gaffuriana 1496/97 Isaac: Missa Carminum

1. Hälfte 16. Jahrhundert: Nationale Komponenten in der Musik um 1500 Josquin Desprez: Vergil-Motetten - Pierre de la Rue: Missae "L'homme armé" um 1500-1530 Cancionero musical de Palacio 1501 Petrucci: Harmonice Musices Odhekaton ca. 1501-1505 Josquin Desprez: Missa "Hercules Dux Ferrariae"

1502 Missae Josquin

1504 Agricola: Missae

1504-14 Petrucci: 11 Bücher Frottole

1506 Isaac: Missae

1507 Isaac: Staatsmotetten zur Eröffnung des Reichstages zu Konstanz

1507-08 4 Bücher Intavolatura di Liuto

1508 Lauden-Drucke in Venedig

um 1510 Liederbuch des Arnt von Aich

1510 Frottolen-Druck in Rom: Canzoni nove

1511 Finck: Missa »In Summis"

- Schlick: Spiegel der Orgelmacher

- Virdung: Musica getutscht

1512 Oeglin: Liederbuch

1512 und 1520 Schlick: Tabulaturen Etlicher Lobgesang

1513-32 Kotter: Tabulaturbuch

1513-36 Schöffer: Liederbücher

um 1515 und 1523 Judenkunig: Lehrbücher zum Lautenspiel

1516 Senfl: Motette »Sancte pater"

1517 Andrea Antico da Montona: Frottole intabulate

- Ornitoparchus: Musicae activae micrologus

1519 Rhaw: Missa »De Sancto Spiritu"

1519/1520 Auflösung der Hofkapelle Kaiser Maximilians I.

1520 Josquin Desprez: Aucunes chansons nouvelles

- Liber selectarum cantionum

um 1520 Buchner: Fundamentbuch

vor 1521/24 Josquin Desprez: Missae "Pange lingua" und "Da pacem"

um 1523 Cavazzoni: Recerchari etc. für Orgel 134 um 1523/24 Senfl: „Lust hab' ich ghabt zuer Musica"

1524 Kleber: Tabulaturbuch

— J. Walter d. Ä.: Geystliche gesangk Buchleyn

1524-26 Stoltzer: Psalm-Motetten nach Luthers Übersetzung

um 1525 Sicher: Tabulaturbuch

1527 Willaert wird Kapellmeister an San Marco in Venedig
67

68

68

68

69

57

69

69

70

70

34 vor 1528 Taverner: Missa »Gloria tibi Trinitas" 90 um 1528 Janequin: Chansons 91 1528 Attaingnant: Chansons nouvelles $\quad 90$ 1529 Agricola: Musica instrumentalis deudsch 136 - Attaingnant: Lautentabulaturbuch 136 vor 1530 Senfl: Missa super "L'homme armé" 79 - Senfl: Missa super »Nisi dominus« $\quad 80$ - Senfl: Marien-Motetten $\quad 80$ 1530 Madrigali di diversi Musici 91 um 1531 Attaingnant: Drucke mit Orgelmusik 136 - Attaingnant: Tanzsammlung für Cembalo 137 1534 und 1544 Ott: Liederbücher

1535 Egenolff: Gassenhawerlin und Reutterliedlin 81

- Milán: Tabulaturbuch "El Maestro" 137

- Verdelot: 1. Buch vierstimmiger Madrigale 92

1535 und 1542/43 Ganassi: Lehrbücher für Flöte und Viola

1536 Francesco da Milano: Lautentabulatur 137

- Willaert: Intavolierungen von Madrigalen Verdelots

1536-49 Neusidler: Lautenbücher

1537 Canzone Villanesche alla Napolitana 92

1538 Narváez: Delphin de musica 139

vor 1539 Arcadelt: 1 . Buch vierstimmiger Madrigale

1539 Hofhaimer und Senfl: Harmoniae poeticae

- Willaert: 1. Buch vierstimmiger Motetten

1539-56 Forster: Liederbücher

um 1540 Johannes von Lublin: Orgeltabulatur

1542 Cipriano de Rore: 1. Buch fünfstimmiger Madrigale

1542 Rhaw: Sacrorum Hymnorum Liber primus

1543 Cavazzoni: Tabulaturdrucke

1543-44 Resinarius: Responsorien und Choralbearbeitungen

1544 Cipriani... Motectae

- Cristóbal de Morales: Missarum libri I, II

- J. Walter d. Ä.: Psalmmotette zur Einweihung der ev. Schloßkirche in Torgau 83

1545 Sixt Dietrich: Hymnen

- Willaert: Canzone Villanesche alla Napolitana

1545-63 Trienter Konzil

1546 Willaert: 1 . Buch fünfstimmiger Madrigale

1547 Clareanus: Dodekachordon

- Othmayr: Symbola Illustrissimorum Principum

um 1550 Orgeltabulatur aus Rovato

- Die Römische Schule

- Tallis: Short Service

1550 Willaert: Salmi spezzati 
um 1550-um 1650 Familie Tieffenbrucker: Lauten- und Violenmacher

1551 Intabulatura nova

- Le Roy: ler Livre de tabulature

1552 Coclico: Musica reservata

1553 Ortiz: Tratado

nach 1553-um 1610 The Mulliner Book

1554 Festa: Magnificat

- Palestrina: 1. Buch der Messen

1555 Bermudo: Declaración de instrumentos musicales

- Lasso: 1. Buch fünfstimm. Madrigale

- Lasso: Madrigali, Vilanesche, Canzoni francesi e Motetti a 4

- Palestrina: 1 . Buch vierstimmiger geistlicher Madrigale

- Vicentino: L'antica musica ridotta alla moderna prattica

1556 Cancionero de Upsala

- Lasso wird Mitglied der Münchener Hofkapelle

- Lasso: 1. Buch Motetten zu 5 und 6 Stimmen

1556/57 Clemens non Papa: 4 Bücher Souterliedekens

1558 Zarlino: Istitutioni harmoniche

1558-95 Jaches de Wert: 11 Bücher fünfstimmiger Madrigale

vor 1559 Lasso: Prophetiae Sibyllarum

1559 Lasso: Bußpsalmen

- Willaert: Musica nova

- Willaert u.a.: 1. Buch Fantasie, Ricercari, Contrapunti

1560 1. Sammlung von Anthems

1562/63 Palestrina: Missa Papae Marcelli

1563 Dreßler: Musica poetica

- Lasso: Lautentabulaturen

- Palestrina: 1. Buch vierstimm. Motetten

- Schiavetti: Madrigali

1564 Lasso: 1. Buch franz. Chansons a 4

1565 A. Gabrieli: 1. Buch fünfstimmiger Motetten

- Goudimel: Hugenotten-Psalter

- Thomas de Santa María: Arte de tañer Fantasia

1566 und 1573 Merulo: 1. Buch Madrigale und 1. Buch Messen

1567 Lasso: Newe teutsche Liedlein

1567 und 1574 Merulo: Ricercari d'intavolatura d'organo und Ricercari da cantare

1568 V. Galilei: Il Fronimo

1570 Baîf gründet die Académie de Poésie et de Musique

1571 A. Gabrieli: Greghesche et Giustiniane a 3

1571 und 1575 Ammerbach: Orgel- und Instrument-Tabulatur und Ein new kunstlich Tabulaturbuch

1573 Lasso: „Viersprachendruck«

1573-76 Lasso: Patrocinium musices $I-V$

141

141

142

96

142

142

96

96

143

97

97

97

97

98

98

98

98

98

99

99

99

100

143

100

101

101

143

102

102

102

103

103

144

103

103

144

144

103

104

144

104

um 1575-um 1665 Familie Ruckers in

Antwerpen: Cembalobauer

145

1576-79 Regnart: Deutsche Lieder 105,175

1577 Cipriano de Rore: Sämtliche Madrigali $a 4$ in Partitur

105

- de Salinas: De musica libri septem

105

- B. Schmid d. Ä.: Orgel- u. Instrument-

Tabulatur

145

1578 Cabezón: Obras de musica para tecla... 145

1578-79 Palestrina: Messen für Mantua $\quad 106$

um 1580 Camerata Fiorentina $\quad 174$

1580 Marenzio: 1. Buch fünfstimmiger Madrigale

106

1581 Balet comique de la Royne $\quad 146$

- V. Galilei: Dialogo 106

- Philippe de Monte: Madrigale $\quad 106$

1582 V. Galilei: erste Monodien in Florenz 175

1583 A. Gabrieli: Psalmi Davidici a $6 \quad 107$

1584 Marenzio: 1. Buch dreistimmiger Villanelle

1585 Marenzio: 1 . Buch vierstimmiger Madrigale

- Eröffnung des Teatro Olimpico in Vicenza 107 1586 Osiander: Geistliche Lieder und Psalmen a 4

1586-91 Gallus: Opus musicum

1587 A. und G. Gabrieli: Concerti per voci et stromenti

108,146

1587, 1590, 1592 Monteverdi: 1. bis

3. Madrigalbuch

1588 Arbeau: Orchésographie

- Byrd: Psalmes, Sonets \& Songs

- Ingegneri: Responsoria Hebdomadae a 4109

— »Musica transalpina"

1589 Intermedien zur Fürstenhochzeit in Florenz

- A. Gabrieli: Madrigali e Ricercari

1589/90 Gallus: Harmoniae Morales

1589 und 1591 Byrd: Cantiones sacrae I, II 110

vor 1591 und vor 1620 Byrd: "The Battel" und "The Bells"

1591 Gumpelzhaimer: Compendium musicae

1591 und 1594 Gastoldi: Balletti a 5 und $a 3$

1592-1622 Zacconi: Prattica di musica

1593 A. und G. Gabrieli: Intonationi d'organo 147

- Lechner: Historie der Passion

1593 und 1594 Morley: Canzonets und Madrigals

1593-1609 Diruta: Il Transilvano

1594 Lasso: Lagrime di San Pietro

1594-96 und 1611 Gesualdo: 6 Bücher fünfstimm. Madrigale

1595-1597/98 Rinuccini und Peri: Dafne 176

1596 Haßler: Madrigali und Neue

Teutsche Gesang

1596-98 Marenzio am Polnischen Hof 
1597 Dowland: First Booke of Songes or Ayres 113

- G. Gabrieli: Sacrae symphoniae I 114, 148

- Morley: Kompositionslehre

- Vecchi: L'Amfiparnasso

1597 und 1598 Canzonets a 4 und Madrigals a 5 of the best Italian Authors

1598 Banchieri: Madrigalkomödie "La pazzia senile"

1598 und 1609 Wilbye: First Set und Second Set of Madrigals

1598-1604 Merulo: Toccate d'intavolatura d'organo I

um 1600-1610 Blütezeit der altenglischen Lautenmusik

um 1600-1621 Sweelinck in Amsterdam

1600 L'Artusi: Overo delle Imperfettioni della musica moderna

- De'Cavalieri: Rappresentazione di Anima e di Corpo

- Rinuccini und Peri: L'Euridice

1601 G. Caccini: Le Nuove Musiche

- Gesius: Geistl. deutsche Lieder

- Hassler: Lustgarten

- Luzzaschi: Madrigale

- Morley: The Triumphes of Oriana

1602 Guédron: Airs de cour

- Viadana: Cento concerti ecclesiastici

1603 Besardus: Thesaurus harmonicus

1603/1621 "Florilegium Portense"

1604 J. Dowland: Lachrymae

- Haußmann: Neue Intraden

- Lasso: Magnum Opus Musicum

1605 Byrd: Messen

- Monteverdi: 5. Madrigalbuch

- Victoria: Officium defunctorum

1605-19 Prätorius: Musikalische Werke

1606 Burmeister: Musica Poetica

- Lechner: Das Hohelied und Deutsche Sprüche

1607 Monteverdi: L'Orfeo

- Monteverdi: Scherzi musicali

- B. Schmid d. J.: TabulaturBuch

1608 Marco da Gagliano: Dafne

- Monteverdi: L'Arianna

1609 Schein: Venus Kräntzlein

1610 R. Dowland: Varietie of Lute-Lessons

- Monteverdi: Messe und Marien-Vesper

1611 R. Ballard d. J.: 1. Lautenbuch

- Peuerl: Neue Padovanen

1611 oder 1612 Schütz: Italienische Madrigale

1612/13 und 1614 Byrd, Bull, Gibbons: Parthenia

1612/14 Vulpius: Deutsche sonntägliche evangelische Sprüche

1613 Cerone: El Melopeo y Maestro

- Gesualdo: 6 Bücher fünfstimmiger Madrigale in Partitur
1614 Erste Opernaufführung außerhalb Italiens

- Monteverdi: 6. Madrigalbuch

189

190

119

1614-19 Prätorius: Syntagma musicum 190

1151615 G. Gabrieli: Canzoni e Sonates 150

1615 und 1627 Frescobaldi: Toccate e Partite 151

1616 Staden: Harmoniae sacrae

1617 Woltz: Nova Musices Organice Tabulatura

152

1619 Kepler: Harmonices mundi 120

— Monteverdi: 7. Madrigalbuch $\quad 191$

- Schütz: Psalmen Davids

vor 1620 The Fitzwilliam Virginal Book 152

1620 Lukačić: Sacrae Cantiones 192

1621-27 Scheidt: Ludi musici 153

1622 Jelić: Parnassia militia concentuum 192

1623 Franck: Musicalisches Lustgärtlein 192

- Titelouze: Hymnes de L'Eglise pour toucher sur l'orgne

1624 Monteverdi: Il Combattimento di Tancredi e di Clorinda

- Scheidt: Tabulatura nova

1624 und 1627 Steigleder: Tabulaturbücher 154

1625 Francesca Caccini: Ballett-Oper "La liberatione di Ruggiero"

- Scheidemann Organist in Hamburg

1626 Correa de Arauxo: Libro de tientos $\quad 155$

1627 Jarzębski: Canzoni e concerti 155

- Schütz: Daphne

1628 Frescobaldi: Il primo libro delle canzoni 155

1628/1661 Schütz: Der Beckersche Psalter 194

1629 Schütz: Symphoniae sacrae I 195

1631 Demantius: Deutsche Passion 120

1632 Eröffnung des Teatro Barberino in Rom 196

1635 Frescobaldi: Fiori musicali 155

1636 Schütz: Musikalische Exequien $\quad 196$

1636/37 Mersenne: Harmonie universelle 197

1636, 1639 Schütz: Kleine geistliche Konzerte I und II

1637 Froberger Hoforganist in Wien

- Eröffnung des ersten öffentlichen Opernhauses in Venedig

1638 Monteverdi: 8. Madrigalbuch $\quad 199$

1638-50 H. Albert: Arien-Sammlungen $\quad 199$

1639 Hammerschmidt Organist in Zittau 200

1640 Kindermann Organist in Nürnberg 200

1640/41 Monteverdi: Selva morale e spirituale 200

1640-70 Carissimi und die Hochblüte des Oratoriums in Italien

1641 Cavalli: La Didone

- Monteverdi: Il ritorno d'Ulisse in patria

- Selle Kantor in Hamburg 204

189 - Tunder Organist in Lübeck 205

1181642 Monteverdi: L'incoronazione di Poppea 205

1642 und 1644 Eccard und Stobaeus:

119 Preußische Festlieder 
1644 Scheidt: LXX Symphonien auff Concerten-manir

- Staden: Seelewig

- Barbara Strozzi: 1. Madrigalbuch

1646-87 Lully am Hofe Ludwigs XIV.

1647, 1650 Schütz: Symphoniae sacrae

II und III

1648 Schütz: Geistliche Chormusik

209

1649 Cesti: Orontea

1650 Scheidt: Görlitzer Tabulaturbuch

1651 Cesti: L'Alessandro vincitor di se stesso 211 - Playford: The English Dancing Master $\quad 156$ 1652 Böddecker Stiftsorganist in Stuttgart 212 1654 Cesti: Cleopatra um 1655 Gaultier: La rhétorique des dieux 1655 Weckmann Organist in Hamburg 1656 Lully gründet »Les petits violons « 1657 Schütz: Zwölf Geistliche Gesänge 1657, 1667 A. Krieger: Arien und Neue Arien nach 1657 Bernhard: Kompositionslehre um 1660 Dolar: Messen

1660/1662 Cavalli: L'Ercole amante $\quad 216$

1664 Schütz: Weihnachtshistorie 216

1664-74 Lully und Molières: Comédie-Ballets für Versailles

1665/66 Schütz: Passionen

1668 Buxtehude Organist in Lübeck

- Cesti: Il pomo d'oro

1669 Kerll: Delectus Sacrarum Cantionum 220

1670 Chambonnières: Pièces de clavecin 220

- Pezel(ius): Hora decima

1671 Schütz: "Schwanengesang"

1672 Lully: Gründung der Académie royale de musique

1673 Theile: Missae juxta veterem contrapuncti stylum

um 1675 Biber: Mysterien-Sonaten

1678-1738 Oper in Hamburg

1679 Schmelzer Hofkapellmeister in Wien 224

1680 Krieger Hofkapellmeister in Weißenfels 224

1681-1700 Corelli: Sonaten op. 1-5 225

1682 Kusser: Suitensammlung 225

- Muffat: Armonico tributo

- Rosenmüller Hofkapellmeister in Wolfenbüttel

1683 Purcell: Sonaten

1683-1721 Ära Alessandro Scarlatti der Oper in Neapel

1685 Purcell: Krönungsanthem »My Heart is inditing"

1686 Marais: 1. Buch Pièces de viole

1687 Lasso: Missa »Jager»

1689 Bruhns Organist in Husum

- Purcell: Dido and Aeneas

- Steffani: Enrico Leone um 1690 Fischer Hofkapellmeister in Rastatt 1693-1720 Oper am Brühl in Leipzig

1695 Pachelbel Organist in Nürnberg

1698 Böhm Organist in Lüneburg
1698-1704 Charpentier: Kirchenmusik für die Sainte-Chapelle 1700 Kuhnau: Biblische Historien 230

1700-17 Niedt: Musikalische Handleitung 231

1701 Academia Philharmonicorum in

$$
\text { Laibach/Ljubljana }
$$

1702 V. Lübeck Organist in Hamburg 232

1703 Brossard: Dictionnaire 233

1705 Händel: Almira 233

1706-28 Rameau: Pièces de clavecin 234

1707 Händel: Dixit Dominus 234

- Händel: Rodrigo 234

1707-12 Bach: Frühe Kirchenkantaten 235

1708 J. G. Walther: Praecepta der Musicalischen Composition 236

1708-17 Bach: Weimarer Orgelwerke 236

1708-1717 (1740) Bach: Orgelbüchlein 237

1709 Händel: Agrippina 238

- Kärntertortheater in Wien 238

1710 Händel Hofkapellmeister in Hannover 239

1711 Händel: Rinaldo 239

1711 und 1728 Heinichen: Generalbaßschule 241

1712 Graupner Hofkapellmeister in Darmstadt 242

— Vivaldi: L'Estro armonico 241

1713 Händel: Utrecht Tedeum 243

1713-30 Fr. Couperin: Pièces de clavecin $\quad 243$

1714 Corelli: Concerti grossi op. $6 \quad 244$

1714-16 Bach: Kirchenkantaten der

Weimarer Zeit

1715-36 Händel: „Wassermusik« 245

1716 Buttstett: Traktat »Ut Mi Sol...« 245

1717-23 Bach: Kantaten der Köthener Zeit 246

1718 Händel: Acis and Galatea 247

1718-21 Bach: Brandenburgische Konzerte 247

um 1718-um 1725 Bach: OrchesterOuvertüren

1719 Brockes: „Der für die Sünde der Welt gemarterte und sterbende Jesus « in verschiedenen Vertonungen

- Stölzel Hofkapellmeister in Gotha

1719, 1729 Händels 1. und 2. Royal Academy of Music

um 1720 Bach: Sonaten und Partiten für Violine Solo

- Bach: Suiten für Violoncello Solo

- Bach: Violinkonzerte

- Zelenka: Orchesterwerke

1720 Händel: Cembalo-Suiten unter Zelenka 252

1721 Telemann Musikdirektor in Hamburg 253

1722 Bach: Das Wohltemperierte Klavier I 255

- J. F. Fasch Hofkapellmeister in Zerbst 258

1722, 1726 Fr. Couperin: Concerts Royaux, Nouveaux Concerts

1722-60 Rameau: Musiktheoretische 
1723-24 Bach: Leipziger Kirchenkantaten, Jahrgang I

1723 ff. Telemann: Kapitänsmusiken

1723-34 (?) Bach: Motetten

1723-40 Bach: Weltliche Kantaten der Leipziger Zeit

1724 Bach: Johannespassion

1724-25 Bach: Leipziger Kirchenkantaten, Jahrgang II

1724-27 Händel: Deutsche Arien

1724-40 Bach: Kirchenkantaten außerhalb der Leipziger Jahrgänge

um 1725 Vivaldi: Die vier Jahreszeiten

1725 Bach: Kantate 127 »Herr Jesu Christ, wahr' Mensch und Gott"

- Fux: Gradus ad Parnassum

- Telemann: Pimpinone

1725/26 Telemann: Der harmonische Gottesdienst

1725-27 Bach: Leipziger Kirchenkantaten, Jahrgang III

1727 Händel: Coronation Anthems

1728 Pepusch und Gay: The Beggar's Opera

1728-29 Bach: Leipziger Kirchenkantaten, Jahrgänge IV und $\mathrm{V}$

1728, 1732 Telemann: Methodische Sonaten

1729 Bach: Matthäuspassion

um 1730 Bach: Klavierkonzerte

- Sammartini: Sinfonien

1730 Händel: Partenope

1731 Bach: Klavierübung I

1731-63 Ära Hasse der Barockoper in Dresden

1732 J. G. Walther: Musicalisches Lexikon

1732-52 Händel: Die großen Oratorien

1733 Bach: h-moll-Messe

- Pergolesi: La serva padrona

- Telemann: Tafelmusik

1733 ff. Händel: Orgelkonzerte

1733-46 Rathgeber: Augsburger Tafelconfect

1733-60 Rameau: Opern und Ballette

1733-64 W. F. Bach Organist in Dresden und Halle

1734-um 1738 Bach: Oratorien zu Weihnachten, Ostern und Himmelfahrt

1735 Bach: Klavierübung 2

- Pergolesi: L'Olimpiade

1736 Pergolesi: Stabat mater

- Sperontes: Singende Muse an der Pleiße

1736, 1739 Händel: Alexanderfest und Cäcilienode

1736-1800 Ära der Barockoper in St. Petersburg

1737 Bach: Klavierübung 3

1737-40 Scheibe: Critischer Musikus

1737-50 Bach: späte Choralbearbeitungen um 1738/39 Bach: kleine lateinische Messen 1738-42 Bach: Das Wohltemperierte Klavier II 1738-57 D. Scarlatti: Cembalo-Sonaten
266
1738-67 C. Ph. E. Bach Cembalist bei Friedrich dem Großen 1739 Händel: Israel in Ägypten 325

1739 und 1740 Mattheson: Der vollkommene Capellmeister und Grundlage einer Ehren-Pforte

um 1740 Monn: Sinfonien

1740 Händel: 12 Concerti grossi op. $6 \quad 290$

1741 Händel: Deidamia 291

wohl 1742 Bach: Goldberg-Variationen 292

1742 Händel: Messias

1742 Eröffnung des neuen königlichen

Opernhauses in Berlin

1742-87 C. Ph. E. Bach: Klaviermusik

1746/47 Bach: Canonische Veränderungen „Vom Himmel hoch"

1747 Bach: Das Musikalische Opfer

- Forqueray: Piecès de viole

1749 Händel: "Feuerwerksmusik"

1749/50 Bach: Die Kunst der Fuge

um 1750 Ostracher Liederbuch

- Tartini: Violinsonaten und -konzerte

1750-78 Marpurg: Musiktheoretische und kritische Schriften

1751 Geminiani: Violinschule

1751-59 d'Alembert: Musikartikel in Diderots Encyclopédie

1752 Händel: Jephtha

- Quantz: Flötenschule

- Rousseau: Le Devin du village

- Standfuß: Der Teufel ist los

1752-68 Riepel: Anfangsgründe zur musikalischen Satzkunst

1753 Beginn der Berliner Liederschule

1753/1762 C. Ph. E. Bach: Versuch über die wahre Art, das Clavier zu spielen

1753-69 Ära Jommelli der Barockoper in Stuttgart

1754/55 Galuppi: Il filosofo di campagna 335

um 1755 Haydn: Streichquartette op. 1 bis op. 3400

- Wagenseil: Sinfonien

1755 Graun: Der Tod Jesu

- Friedrich II. von Preußen und C. H. Graun: Oper "Montezuma"

1756 L. Mozart: Violinschule

um 1757 J. Stamitz: Sinfonien

1757 Kirnberger: Anleitung mit Würfeln zu komponieren

1758 Adlung: Anleitung zu der musikalischen Gelahrtheit

seit 1759 Haydn: Erste Sinfonien 402

um 1760 Ivančič: Sinfonien 338

- Sorkočević: Sinfonien 338

1760 Holzhauer: La Betulia liberata 338

1760-79 J. Chr. Bach: Opere Serie 338

1761 The Noblemen's Catch Club in London 339

1761-90 Haydn Kapellmeister bei den

Fürsten Esterházy

1762 Gluck: Orfeo ed Euridice 
1762-91 Haydn: Opern

1764-86 Mozart: Sinfonien

1765 Neues Theater in Laibach/Ljubljana

1765 ff. Haydn: Klavierwerke

1765-75 Haydn: Kompositionen für Baryton

1767 Gluck: Alceste

- J. A. Hiller: Lottchen am Hofe

1767/68 Rousseau: Dictionnaire de musique

1768 Haydn: »Applausus-Kantate"

1768-88 C. Ph. E. Bach Musikdirektor

in Hamburg

1768-1803 Grétry: Opern

1769 Gaßmann: »Opera seria»

1770 Gluck: Paride ed Elena

1771-79 Kirnberger: Die Kunst des reinen Satzes

1772 Haydn: Streichquartette op. 20

1772 ff. Mozart: Klavierwerke

1773 Schweitzer: Alceste

1773-75 Mozart: Violinkonzerte

1774 Gluck: Iphigénie en Aulide

1775 G. A. Benda: Melodramen

1777 Gluck: Armide

- Holzbauer: Günther von Schwarzburg

1778 Kamieński: Glück im Unglück

- Umlauf: Die Bergknappen

1778/79 Herder: Volksliedersammlungen

1779 Gluck: Iphigénie en Tauride

- Mozart: Krönungsmesse

1781 Haydn: Streichquartette op. 33

- J. A. Hiller Gewandhauskapellmeister in Leipzig

- Mozart: Idomeneo

- Reichardt: Frohe Lieder für deutsche Männer

1781-84 Mozart: Die großen Bläserserenaden

1782 Mozart: Entführung

- Schulz: Lieder im Volkston

1782/83 Mozart: $c$-moll-Messe

1782-85 Mozart: »Haydn-Quartette»

1782-93 Koch: Versuch einer Anleitung zur Komposition

1783 Albertini: Don Juan albo

- Haydn: Violoncellokonzert

1784 Paisiello: Il Rè Teodoro

- Salieri: Les Danaides

- École Royale de Chant et de Déclamation

1785/86 Haydn: Pariser Sinfonien

1786 Dittersdorf: Doktor und Apotheker

- Haydn: Instrumentalmusik über die Sieben Worte

- Mozart: Figaro

- Mozart: Streichquartett D-dur (KV 499)

- Mozart: Konzertarie KV 505

1787 Mozart: Don Giovanni

1787 und 1795/96 Heinse: Ardinghello und Hildegard von Hohenthal

1787-91 Mozart: verschiedene Quintette

1788 Mozart: die 3 letzten Sinfonien
1788-1801 Forkel: »Musikgeschichte"

1789/90 Mozart: die sog. Preußischen

Streichquartette

1789-94 Die Pariser Oper in der französischen

Revolution

um 1790 Betscher: Kirchenmusik in Rot an der Rot

360

1790 Albrechtsberger: Kompositionslehre $\quad 360$

1790 Gossec: Te deum 361

- Mozart: Cosi fan tutte $\quad 428$

- Novak: Der tolle Tag 361

1791 Gründung der Singakademie in Berlin 362

- Mozart: Zauberflöte 429

- Mozart: Titus 433

- Mozart: Requiem

431

1791-95 Haydn: Londoner Sinfonien $\quad 434$

1791-1805 Zumsteeg: Balladen und Lieder 362

1792 Cimarosa: Il matrimonio segreto 363

- Rouget de l'Isle: La Marseillaise 363

1792-97 Viotti: Violinkonzerte 364

1793 Lesueur: La Caverne 364

1794 Philharmonische Gesellschaft

in Laibach/Ljubljana

364

1795 Altenburg: Trompeterschule $\quad 300$

- Körner: Über Charakterdarstellung in der Musik

438

394

364

- Schenk: Der Dorfbarbier $\quad 365$

1796-1802 Haydn: Die großen Messen 439

1797 Cherubini: Médée

365

440

- Wackenroder und Tieck: Herzensergießungen

366

1798 Haydn: Die Schöpfung 441

- Kauer: Das Donauweibchen 367

1798-1800 Beethoven: Streichquartette op. 18443

1798-1848 Allgemeine Musikalische Zeitung in Leipzig 368

1799-1802 Beethoven: 1. und 2. Sinfonie 445

1799-1824 Elsner Kapellmeister in Warschau 368

um 1800 Bortnjansky: "Ich bete an die Macht der Liebe"

1801 Haydn: Die Jahreszeiten

- Triest: Bemerkungen über die Ausbildung der Tonkunst in Deutschland im

18. Jahrhundert

$1801 \mathrm{ff}$. Musikvereine und Musikfeste

in Deutschland

1802 Forkel: Bach-Biographie $\quad 517$

421 - Koch: Musikalisches Lexikon 447

422 1802-03 Beethoven: "Kreutzer-Sonate" 447

4221803 Beethoven: 3. Sinfonie 449

4231804 Ivan Jarnović gestorben in St. Petersburg 518

- Paër: Oper »Leonore" 518

1804/05 Jean Paul: Flegeljahre 518

1804-08 Beethoven: 5. Sinfonie 451

1804-10 Cramer: Klavier-Etüden 518 
1805 Nägeli gründet das Zürcherische Singinstitut

1805-08 Arnim und Brentano: Des Knaben Wunderhorn

1805/1814 Beethoven: Fidelio

1806 Beethoven: Streichquartette op. 59

- Beethoven: Violinkonzert

- Schubart: Ideen zu einer Ästhetik der Tonkunst (1784)

1806, 1811/12, 1812 Beethoven: 4., 7. und 8. Sinfonie

seit 1806 Onslow: Streichquartette und -quintette

1807 Méhul: Joseph

- Spontini: La Vestale

1807/08 Beethoven: 6. Sinfonie

seit 1807 Akademie-Konzerte in Mannheim

1809 E. Th. A. Hoffmann: Ritter Gluck

1809, 1810, 1811 Beethoven: Streichquartette op. 74 und op. 95 und Klaviertrio op. 97

1810 E. Th. A. Hoffmann: Besprechung der fünften Sinfonie von Beethoven

- Erstes deutsches Musikfest in Frankenhausen/Thüringen

1811-16 Schubert: Frühe Balladen

1812 Mälzel: Erfindung des Metronoms

- Gründung der Gesellschaft der Musikfreunde in Wien

1813 G. Rossini: L'Italiana in Algeri

1813-1817/18 Schubert: 6 frühe Sinfonien

1814 Field: Nocturnes

1815 Pamer: Wiener Walzer

- Schubert: Erlkönig

19. Oktober 1815 Schubert: Gretchen am Spinnrade

1815 ff. Schubert: Klavierwerke

1816 Beethoven: An die ferne Geliebte

- E. Th. A. Hoffmann: Undine

- Hummel: Klavierseptett und -quintett

- Rossini: Il barbiere di Siviglia

1817-26 Clementi: Gradus ad Parnassum

ca. 1818 Reicha: Cours de Composition

1818 Gruber: „Stille Nacht, heilige Nacht»

1818-66 Loewe: Balladen

nach 1818 Schubert: zweite Epoche seines Liedschaffens

1819 Louise Reichardt gründet in Hamburg eine Musikschule für Frauen

- Schubert: »Forellenquintett"

- Spontini: Olympie

1819 und 1821 Weber: Aufforderung zum Tanz und Konzertstück

1819-23 Beethoven: Missa solemnis

1819/22, 1828 Schubert: Messen in As und Es 1820 Paganini: 24 Caprici

- Paris: Assurence Succès Dramatique

- Schneider: Das Weltgericht

- Schubert: Quartettsatz c-moll

- Schubert: Die Zauberharfe
1820-45 Berwald: Symphonien

1821 Schubert beginnt Lieder $\mathrm{zu}$ veröffentlichen

521

454

457

458

522

- Schubert: Gesang der Geister über den Wassern

479

- Weber: Der Freischütz

539

1821/22 Schubert: Alfonso und Estrella 543

1821-28 »Schubertiaden« in Wien

480

1822 Livadić: Klaviermusik und Lieder $\quad 544$

- Schubert: Sinfonie h-moll

481

- Zöllner gründet in Leipzig ein »Musikalisches Institut «

1822-24 Beethoven: 9. Sinfonie

482

1822-26 Beethoven: Die letzten Streichquartette

1823 Schubert: Fierabras

1823 und 1826 Weber: Euryanthe und Oberon

1823/24 Schubert: Die schöne Müllerin

1824, 1826 Schubert: Streichquartette a-moll, d-moll, $G$-dur

1825 Boieldieu: Die weiße Dame

- Schubert: Große C-dur-Sinfonie

1825/26 und 1836 Moscheles: Klavier-Etüden 548

1825-49 Chopin: Klavierwerke 548

1826 Hérold: Oper »Marie» 550

- Mendelssohn: Ouvertüre "Ein Sommernachtstraum"

1826/27 Gesellschaft zur Aufführung von Schauspielen und Opern in Ljubljana

1826-60 Silcher: 12 Hefte Volkslieder für 4 Männerstimmen gesetzt

1826-86 Liszt: Klavierwerke

1827 Donizetti: Le convenienze ed inconvenienze teatrali

- Schubert: Winterreise

- Erstes deutsches Sängerfest in Plochingen/ Württemberg

- Gründung des Musikvereins in Zagreb $\quad 554$

1827, 1828 Schubert: Klaviertrios Es-dur und B-dur

1828 Auber: La muette de Portici

- Felix und Fanny Mendelssohn: Lieder op. 8557

- Paganini in Wien

- Schubert: Streichquintett C-dur

- Schubert: »Schwanengesang"

1829 Kiesewetter: Die Verdienste der Niederlaender um die Tonkunst

- Lanner Hofballmusikdirektor in Wien

- Wiederaufführung von Bachs Matthäuspassion durch Mendelssohn in Berlin 560

- Rossini: Guillaume Tell

1829-32 Mendelssohn: Lieder ohne Worte 561

um 1830 Czerny: Schule der Geläufigkeit $\quad 562$

1830 Auber: Fra Diavolo

563

- Berlioz: Symphonie fantastique 563

1831 Bellini: Norma $\quad 565$

1833 Marschner: Hans Heiling 566 
- Nestroy: Der böse Geist Lumpazi Vagabundus

1834 Kreutzer: Das Nachtlager von Granada

- Schumann gründet die Neue Zeitschrift für Musik

1835 Halévy: Die Jüdin

1835-44 Fétis: Biographie universelle

1836 Adam: Der Postillon von Lonjumeau

- Glinka: Ein Leben für den Zaren

- Meyerbeer: Die Hugenotten

1836-1838/39 Schumann: Kinderszenen und C-dur-Fantasie

1836, 1846 Mendelssohn: Paulus und Elias

1837 Lortzing: Zar und Zimmermann

1837-47 A. B. Marx: Kompositionslehre

1840 Gründung der Musical Antiquarian Society in London

- Schumann: »Lieder-Jahr«

1840-83 Vieuxtemps: Violinkonzerte

1841-45 und 1850 Schumann: Klavierkonzert und Violoncellokonzert

1841-51 Schumann: Symphonien

1842 Mendelssohn: Schottische Symphonie

- Verdi: Nabucco

1842-71 Gade: Symphonien

seit 1842 Wiener Philharmonische Konzerte

1843 Donizetti: Don Pasquale

- Wagner: Holländer

- Gründung des Konservatoriums in Leipzig

1843 und 1850 Schumann: Das Paradies und die Peri und Genoveva

1844 Berlioz: Instrumentationslehre

1845 Wagner: Tannhäuser

1845 ff. Gottschalk: Klavierstücke

1846 Lisinski: Ljuba $i$ zloba

1846-1850 Fanny Hensel: Ausgewählte Werke 585

1847 Flotow: Martha

- Clara Schumann: Klaviertrio

- Verdi: Macbeth

1848 Glinka: Kamarinskaja

- J. Strauß (Vater): Radetzky-Marsch 588

1848/58 Moniuszko: Halka

1848-1861 Liszt in Weimar. Die Neudeutschen und ihre Symphonischen Dichtungen $\quad 590$

1849 Nicolai: Die lustigen Weiber

591

1850 Wagner: Lohengrin

1850-1886 A. Rubinstein: Symphonien und Klavierkonzerte

591

592

1851 Verdi: Rigoletto

592

1852/59 Gounod: »Ave Maria"

593

1852-1896 Brahms: Lieder

1853 Verdi: Il Trovatore und La Traviata

1853-1864 Proske: »Musica Divina»

1853-1893 Brahms: Klavierwerke

1854 Ernst: "Le Roi des Aules"

- Hanslick: Vom Musikalisch-Schönen

1856 Badarzewska-Baranowska: „Gebet einer Jungfrau"

594

594

595

595
- Cornelius: Weihnachtslieder

- Erk: Deutscher Liederhort

600

- Dargomyschski: Russalka und

Der steinerne Gast

600

seit 1857 Gürzenich-Konzerte in Köln 601

1858 Lahrer Commersbuch 601

- Offenbach: Orphée aux Enfers 601

1858-1887 Brahms: Solokonzerte 602

1859 Gounod: Faust 603

1860 Suppè: Das Pensionat 603

1861-68 Brahms: Ein deutsches Requiem 604

1861-74 Farrenc: Le Trésor des pianistes $\quad 604$

1862 Wieniawski: Violinkonzert 604

- Gründung des Konservatoriums in St. Petersburg

605

1864-1868 Bruckner: Messen 605

1864 Offenbach: Die schöne Helena 601

1865 Liszt: Die Heilige Elisabeth 606

- Wagner: Tristan und Isolde 607

1866 Bruch: Violinkonzert g-moll 608

- Gründung des Konservatoriums in Moskau 605

- Smetana: Die verkaufte Braut 608

- Thomas: Mignon 609

1866-68 Ambros: "Musikgeschichte» 609

1867 J. Strauß (Sohn): An der schönen blauen Donau

609

- Verdi: Don Carlos

610

1867-1901 Grieg: Lyrische Stücke

611

seit 1867 Balakirew-Kreis (Gruppe der

»Fün $«)$ in St. Petersburg

611

1868 Eitner gründet die erste Gesellschaft für Musikforschung

- Wagner: Meistersinger

612

612

$\begin{array}{ll}\text { 1868-1877 Mussorgski: Liederzyklen } & 613 \\ \text { 1868-1876 Freundschaft Wagner-Nietzsche } & 613\end{array}$

1869-76 Wagner: Der Ring des Nibelungen 615

1870 Delibes: Coppelia

- Zajc: Mislav

618

618

seit 1870/1901 Urheberrechtsgesetzgebung und Vertretungsgesellschaften

662

1871-1902 Davorin Jenko Dirigent des

Serbischen Nationaltheaters in Belgrad $\quad 618$

1871 Verdi: Aida

1871/1888 Die Internationale

1872/73 Bruckner: 3. Symphonie

1872-79 Smetana: Mein Vaterland

1873 Lalo: Symphonie espagnol

1874 Bruckner: 4. Symphonie

- Mussorgski: Boris Godunow

618

620

620

622

623

623

624

625

626

- Verdi: Requiem

626

1874 J. Strauß (Sohn): Die Fledermaus

626

1874/75 Grieg: Musik zu Ibsens Peer Gynt

1874-1893 Tschaikowsky: Symphonien

und Konzerte

627

1875 Bizet: Carmen

628

630

1876-1885 Brahms: Symphonien 630

5971877 Saint Saëns: Samson et Dalila 632 
1878 J. J. Abert: Ekkehard

1878/79 Bruckner: Streichquintett

1878-81 Kuhač: Sammlung Südslawischer Volkslieder

seit 1878/1887 Grammophon und Schallplatte

1879 Bruch: Das Lied von der Glocke

1879 Suppè: Boccaccio

- Tschaikowsky: Eugen Onegin

1881 Offenbach: Hoffmanns Erzählungen

1881/83 Bruckner: 7. Symphonie

1881/84 Bruckner: Te Deum

1881-1910 Glasunow: Symphonien

1882 Wagner: Parsifal

seit 1882 Berliner Philharmoniker

1882/83, 1894/95 R. Strauss: Lieder op. 10 und $o p .29$

1883-1909 Stevan Mokranjac: Rukoveti

1884 Franz: Liederheft op. 52

1884-87 Bruckner: 8. Symphonie

1885 Anthologie »Als der Großvater die Großmutter nahm "

- Franck: Variations symphoniques und Prélude, Choral et Fugue

- J. Strauß (Sohn): Der Zigeunerbaron

- Sullivan: The Mikado

1887 Verdi: Otello

1888 Rimski-Korsakow: Scheherazade

- Wolf: Mörike-Lieder

seit 1888 Concertgebouw-Orchester Amsterdam

1889 R. Strauss: Tondichtung "Don Juan «

1889-1912 Mahler: Symphonien

1890 Borodin: Fürst Igor

- Mascagni: Cavalleria rusticana

seit 1890 Beljajew-Kreis in St. Petersburg

1891-1894 Brahms: späte Kammermusik

1891/94 Bruckner: 9. Symphonie

1891-1927 Rachmaninow: Klavierkonzerte

1891/92-1924/25 Nielsen: Symphonien

1892 Leoncavallo: Der Bajazzo

- Massenet: Werther

1892-1900 Granados: Danzas espagnolas

1892-1913 Skrjabin: Klaviersonaten

seit 1892 Denkmäler Deutscher Tonkunst

1893 Dvořák: Symphonie »Aus der

Neuen Welt

632

632

633

663

633

603

633

633

633

634

634

635

636

636

637

637

637

638

638

626

638

639

640

640

641

641

663

642

642

611

643

644

644

644

642

645

645

664

645

646

647

- Humperdinck: Hänsel und Grete

- Riemann: Lehre von den tonalen Funktionen der Akkorde

- Verdi: Falstaff

1894 Debussy: Prélude à l'après-midi d'un faune

seit 1894 Denkmäler der Tonkunst in Österreich

1896 Fibich: Klavierstück »Poème"

1896-1920 Fauré Lehrer am Conservatoire in Paris

1897 Dukas: Der Zauberlehrling

645

648

649

649
1898-1900 Reger: Die großen Choralphantasien für Orgel 665

1899 Lincke: Frau Luna 665

- Schönberg: Verklärte Nacht 665

1900 Elgar: Oratorium »The Dream of Gerontius"

649

- Puccini: Tosca

649

1901 Gründung der Warschauer Philharmonie und »Das junge Polen «

666

1901-1917 Ravel: Klavierwerke 666

1902 Cilèa: Adriana Lecouvreur $\quad 667$

- Debussy: Pelléas et Mélisande 667

1904 Janáček: Jenufa

- Schillings: Melodram »Das Hexenlied

1904/05 Delius: "Eine Messe des Lebens"

668

650

1904-1913 Riemann: Handbuch

der Musikgeschichte

1905 Lehár: Die lustige Witwe

650

- Sibelius: Violinkonzert

1905, 1909 R. Strauss: Salome und Elektra

1906 Bersa: Oper "Der Eisenhammer"

- Kaiserliederbuch

seit 1906 Bartók betreibt vergleichende Musikfolklore

1908 Albéniz: Iberia-Suite

- Ives: The Unanswered Question

- Skrjabin: Le Poème de l'Extase

- Gründung der Oper in Sofia/Bulgarien

1908/09 Schönberg: George-Lieder

1908-1939 Bartók: Klavier-Lehrwerke

- Bartók: Streichquartette

650

669

651

670

670

651

699

652

671

672

673

673

700

1909 Diaghilew gründet »Ballets Russes « 674

- Schönberg: Fünf Orchesterstücke op. 16674

- Der Zupfgeigenhansl und die

Jugendmusikbewegung

1910 Strawinsky: Feuervogel

1910-13 Debussy: Préludes I, II

675

676

676

676

652

677

- Strawinsky: Petruschka

1911/1918 Bartók: Oper »Herzog

Blaubarts Burg"

677

$\begin{array}{ll}1912 \text { Mahler: 9. Symphonie } & 677 \\ 1913 \text { Lili Boulanger erhält den Prix de Rome } & 678\end{array}$

- Strawinsky: Le Sacre du Printemps 678

1914 Reger: Mozart-Variationen

679

1916 (1907) Busoni: Entwurf einer neuen Ästhetik der Tonkunst

1916 Korngold: Der Ring des Polykrates und Violanta

701

- Das sog. Historische Symphoniekonzert in Zagreb

1916/17 Prokofieff: „Symphonie classique»

1916, 1933 Szymanowski: Violinkonzerte

704

680

1917 Pfitzner: Palestrina

652

1917, 1924, 1929 Respighi: Fontane di Roma, Pini di Roma, Feste romane

680

1918 Dora Pejačević: Symphonie fis-moll, op. 41 
- Die Gruppe »Les Six « tritt an die Öffentlichkeit

1919 de Falla: Der Dreispitz

704

681

1920/22 Gründung der Musikakademie in Zagreb/Kroatien

seit 1920 Salzburger Festspiele

seit den 1920er Jahren Weltweite

Verbreitung des Jazz

1921-27 Hindemith: "Kammermusiken"

seit 1921 Donaueschinger Musiktage

1922/23 Sammelbände für vergleichende

Musikwissenschaft

1922, 1924 Musik des Mittelalters in Karlsruhe und in Hamburg

1923 Honegger: „Pacific 231"

1924 Gershwin: Musical "Lady Be Good"

- Gershwin: "Rhapsody in Blue"

- Romberg: The Student Prince

- Slavenski: 1. Streichquartett

1924/25-1971 Schostakowitsch: Symphonien

1925 Berg: Wozzeck

1925/27 Varèse: Arcana

1926 »Vereinigung der jungen polnischen

Musiker in Paris «

1927 Cotovac: Symphonischer Kolo

- Křenek: Jonny spielt auf

- Wiederaufführung von Perotins Organum "Sederunt principes"

- Raymond: "Ich hab' mein Herz in Heidelberg verloren "

- Strawinsky: Oedipus Rex

1927/30 Weill/Brecht: Mahagonny

1927-1943 Webern: Zwölftönige Werke op. 20 bis op. 31

1928 Weill/Brecht: Die Greigroschenoper

- Ravel: Boléro

- Schönberg: Orchestervariationen op. 31

1928/1935 Berg: Lulu (Fragment)

1929 Kogoj: Oper »Die schwarzen Masken

- Osterc: Suite für Orchester

1929/31 Varèse: Ionisation

1929-1949 Eisler: Politische Songs und Lieder

1930 Strawinsky: Psalmensymphonie

seit 1930 Orff-Schulwerk

1931 Konjović: Oper »Koštana»

1931/32 Prokofieff: 5. Klavierkonzert

1933 "Gesellige Zeit"

1933-1945 Schlager, Tanz- und Filmmusiken im »Dritten Reich«

1935 Berg: Violinkonzert

- Gotovac: Oper "Ero der Schelm"

1935/36 Heidegger: Vom Ursprung des Kunstwerkes

1936 Barber: Adagio für Streicher

706

707

707

708

708

709

709

710

710

710

711

713

713

714

714

714

715

715

717

718

720

722

722

711

725

725

713

726

726

728

729

730

731

731

732

734

734

734
- Bartók: Musik für Saiteninstrumente, Schlagzeug und Celesta

734

736

737

- Erster Tonbandmitschnitt eines Konzertes

1936-1962 Hartmann: Symphonien

737

6811937 Hindemith: Unterweisung im Tonsatz 737

- Glenn Miller gründet seine Big Band

1938 Schmidt: Oratorium »Das Buch mit sieben Siegeln"

739

- Ausstellung »Entartete Musik«

654

739

739

- Hindemith: Mathis der Maler

1938/39 Distler: Mörike-Chorliederbuch

741

1942 Schaeffer gründet in Paris ein akustisches Versuchsstudio

1943 Rodgers: Musical „Oklahoma! «

1946 Švara: Veronika Deseniška

742

1946-48 Messiaen: Turangalîla-Symphonie

742

seit 1946 Ferienkurse für Neue Musik in

Kranichstein

758

1947 Thomas Mann: Doktor Faustus 743

1949 Orff: Antigonae

759

seit 1949 »Urtextbewegung «

seit 1950 Neue historisch-kritische

Musiker-Gesamtausgaben

761

1951 Dessau: Lukullus

761

- Eimert gründet das 1. elektronische Studio in Köln

743

- Strawinsky: The Rake's Progress

762

763

1952 Henze: Boulevard Solitude

- Liebermann: Leonore 40/45

1953 Blacher: Abstrakte Oper Nr. 1

1956 Loewe: Musical "My Fair Lady"

764

764

744

765

765

- Stockhausen: Gesang der Jünglinge $\quad 766$

seit 1956 »Warschauer Herbst火 767

1957 Bernstein: Musical »West Side Story« 767

- Boulez: Le Marteau sans maître 768

- Fortner: Bluthochzeit 768

- Schönberg: Moses und Aron 769

1958 Cage bei den Darmstädter Ferienkursen 770

- Lutosławski: Trauermusik

771

seit 1960 Rasche Verbreitung von neuen Schallplatten und von Tonbändern

1961 Ligeti: Atmosphère

771

772

- Stockhausen: Klavierstück X

773

seit 1961 Biennale für Zeitgenössische Musik in Zagreb

773

1962 Britten: War-Requiem 773

- Kagel: Sur scène

774

1962-65 Penderecki: Stabat mater und Lukaspassion

775

1965 Zimmermann: Die Soldaten 775

1966 Isang Yun: Orchesterstück »Réak» 776

1967/68 MacDermot: Musical »Hair» 777

1988 Theodorakis: Ballett "Alexis Sorbas« 777 


\section{Vorwort}

Im Grunde genommen sind nur musikalische Werke »Fakten der Musikgeschichte«. Dementsprechend sollte eine »Musikgeschichte « eigentlich nur von diesen berichten. So sind denn auch in diesem Buche meist nur die einzelnen Werke aufgeführt, und zwar in chronologischer Folge. Der Leser findet das Werk, das er sucht, also unter der Jahreszahl der Entstehung (manchmal auch der ersten Drucklegung oder der Uraufführung). Wenn man dieses Jahr nicht weiß, muß man das Werk im Register unter dem Namen des Komponisten suchen, und dann wird man es im Buche an seinem Ort in seiner Zeit finden.

Ein Werk hat seinen Ort aber nicht nur in der Chronologie, sondern auch in Zusammenhängen. Deshalb gehen, jeweils nach Epochen getrennt, den Daten und Werken Abschnitte voraus, in denen historische und gattungsmäßige Zusammenhänge dargestellt sind. Diese Zusammenhänge allerdings sehen sich von verschiedenen Gesichtspunkten aus eventuell verschieden an. In solchen Fällen ist der Historiker dann gezwungen, ein und dieselbe Epoche einmal aus diesem, ein andermal aus jenem $\mathrm{Zu}$ sammenhang zu sehen und zu interpretieren. Dementsprechend ist hier der Zeitraum zwischen rund 1430 und 1600 zweimal behandelt, einmal im Zusammenhang der endgültigen Ausprägung der Vokalpolyphonie und einmal im Zusammenhang der Entstehung der Instrumentalkomposition. Ebenso ist der Zeitraum zwischen rund 1750 und 1830 einmal dargestellt unter dem Gesichtspunkt der früh- und dann der hochromantischen Vorstellung von Musik als Tonsprache und ein zweites Mal als die Zeit der Wiener Klassiker. Und Franz Schubert erscheint mit einem Teil seiner Werke im Zusammenhang der Wiener Klassiker, mit einem andern Teil im Zusammenhang der musikalischen Hochromantik.

Das Prinzip der reinen Chronologie der Werke ist also zweimal durchbrochen. Um das auszugleichen, sind vorne als ein zweites Inhaltsverzeichnis alle verzeichneten Werke in einer Tabelle streng chronologisch zusammengestellt. Diese Tabelle wird auf ihre Weise die erstaunliche Vielfalt der Erscheinungen zeigen und die manchmal noch erstaunlichere Verschiedenheit des Gleichzeitigen.

Der Begriff Werk ist mehrdeutig. Man nennt z. B. jedes der Madrigalbücher von Monteverdi ein Werk, aber auch jedes einzelne Madrigal daraus, oder Beethovens op. 59 und jedes einzelne der drei Streichquartette daraus. Hier sind in der Regel die Opera, also die Sammlungen, verzeichnet und nicht die einzelnen Werke. Im Gegensatz dazu werden aber auch manchmal Einzelwerke zu Gruppen zusammengestellt, wenn dadurch Zusammenhänge und Eigenheiten besser deutlich werden können als bei der Nennung und Beschreibung jedes einzelnen Werks, z.B. bei den Sinfonien Joseph Haydns. Dies verstößt zwar gegen den gebotenen Respekt vor der Individualität des einzelnen Werkes, aber die musikgeschichtliche Zielrichtung dieses Buchs verlangt nun einmal solche Zusammenschlüsse - wie sie auf der anderen Seite verbietet, jedes Werk zu verzeichnen, das einen Platz in der Musikgeschichte hat oder verdient.

Obwohl das Buch sich an den Werken als den eigentlichen Fakten der Musikgeschichte orientiert, findet man bei vielen »Werkartikeln« nur wenig zum Werk selbst. Der Grund liegt nicht in der Schwierigkeit, über Meisterwerke etwas Substanzielles zu sagen - obgleich dies in der Tat schwer ist - sondern darin, daß der Gegenstand des Buches die Musikgeschichte als Zusammenhang ist, und diesen gilt es darzustellen, auch aus der Perspektive der Werke. Freilich, denjenigen, der beim Stichwort »Zusammenhang « nur an »Einflüsse« denkt, den muß ich enttäuschen.

Schließlich: Biographien sowie gattungsgeschichtliche und musiksoziologische Ausführungen als solche fehlen; sie sind in einem, vor allem auf Werke konzentrierten Buch nur schlecht unterzubringen. Trotzdem sind selbstverständlich die notwendigen biographischen Fakten verzeichnet und wird immer wieder auf die gleichsam quer durch die Musikgeschichte laufenden Zusammenhänge, welche die musikalischen Gattungen und die Soziologie der Musik, der Musiker und des Musiklebens stiften, hingewiesen, nur eben jeweils bei den einzelnen »Werkartikeln«.

Die unlösbaren Probleme der riesigen Stoffmenge einerseits und der ungleichen Teilmengen in den verschiedenen Epochen andererseits, aber auch das Problem des unterschiedlichen Interesses der Be- 
nutzer an den einzelnen Epochen, diese Probleme sind hier etwas anders gelöst als sonst in vergleichbaren »Musikgeschichten«. Die Überlegung nämlich, daß allgemein die Kenntnis der neueren Musikgeschichte größer ist als die der älteren, und daß bestimmte historische Fakten und Zusammenhänge wohl bekannt sind, während man von vielen anderen fast nichts weiß, auch die Überlegung, daß jedermann sich über das weithin Bekannte leicht informieren kann aber oft nur schwer über das auch nur ein wenig abseits Liegende - diese Überlegungen haben mich veranlaßt, den Gleichheitsgrundsatz, der ja vielleicht auch in der Geschichtsschreibung gelten könnte, einmal zu verlassen, ja auf Ausgewogenheit in der Berücksichtigung der Fakten zu verzichten: es sollte Platz gewonnen werden für einiges, das wesentlich zur Sache gehört, aber sonst meist nicht oder nicht hinreichend berücksichtigt wird.

Bei den überragenden Persönlichkeiten der Musikgeschichte ist jeweils einmal den Lebensdaten der Geburts- und Sterbeort beigegeben, nämlich dort, wo ihr Name zum ersten Mal im Kopf eines »Werkartikels« vorkommt.

In Werktiteln und Textzitaten ist die Schreibung weder überall original beibehalten noch überall normalisiert. Ein Quellenwerk ist das Buch nicht! Manchmal allerdings steckt hinter einer bestimmten Schreibweise auch eine bestimmte Tendenz des Musikhistorikers. So etwa sind bis in die Zeit der Wiener Klassiker »Sinfonie« und »Fantasie« stets in der Schreibweise der italienischen Gattungstradition geschrieben. Weil das Bewußtsein von dieser aber im 19. Jahrhundert verlorengeht, schreibt man dann vornehmlich »Symphonie» und »Phantasie« - und hinter diesen Bezeichnungen steht eine Weltanschauung, die man orthographisch abbilden sollte.

Auf die wissenschaftliche Diskussion einzelner Fakten und auf Anmerkungen ist verzichtet. Das Buch richtet sich an die Kenner und Liebhaber der Musik. Die Literaturhinweise und das ausführliche Literaturverzeichnis können indessen den Interessierten auf den Weg zur eigenen Weiterarbeit leiten. 